\title{
TARGET REDEMPTION NOTES
}

\author{
Chi Chiu CHU ${ }^{1}$
}

\section{Yue Kuen KWOK ${ }^{23}$}

The target redemption note is an index linked note that provides a guaranteed sum of coupons (target cap) with the possibility of early termination. In a typical structure, the coupons are calculated based on an inverse floating LIBOR / Euribor formula. Once the accumulated amount of coupons has reached the pre-specified target cap, the note will be terminated with final payment of the par. The knock-out criterion depends on a path dependent state variable defined by the running accumulated coupon sum. In some simplified cases, we manage to obtain a closed form valuation formula for the note value. We propose several numerical schemes for pricing the note under the one-factor and two-factor short rate models. Pricing behaviors of the target redemption note are also explored.

\section{INTROUDCTION}

A target redemption note is similar to an inverse floating rate note, embedded with additional features like the possibility of early termination and a guaranteed sum of coupon payments. As an example, let us consider the 5-year target redemption note issued by Credit Suisse First Boston on 10 November, 2003. The first year coupon rate is fixed at $9 \%$. The coupon rates in subsequent years are calculated based on an inverse floating formula, $\max (8.65 \%-2 L, 0)$, where the index $L$ denotes the 12 -month Euribor on the coupon date. The note will be terminated prematurely on a coupon date when the accumulated coupon rate meets the target cap of $15 \%$. The salient feature of the note is that the date of the par payment is uncertain, which is taken to be the earlier date among the pre-specified note's maturity date and the coupon date when the accumulated coupon amount meets the target cap. The lure of a handsome initial coupon combined with the perception that the par may be received within a short span of time has made these notes attractive to Asian retail investors in the early 2000's when the interest rates were at a low level. Obviously, the investor has a higher gain on the time value of the cash flow stream when the Euribor decreases since a shorter time is required to collect the coupon payments and par. At the other extreme, the investor faces the worst scenario when the 12-month Euribor trades above $4.325 \%$ one year later and never comes down again. In this case, he then has to hold the note for 5 years and receive the par and the remaining coupon on the maturity date.

The note value is given by the sum of present values of the par and coupon payments and this sum depends on the times at which the payments are received by the note holder. The interest rate fluctuation leads to uncertainty in the coupon payments received on the coupon dates, and also results in uncertainty in the redemption date of the note (knock-out). The uncertainty regarding the termination date is governed by a path dependent variable, which is the running accumulated coupon sum. The note

\footnotetext{
${ }^{1}$ Chi Chiu Chu is in the Department of Statistics, University of Toronto, Toronto, Ontario M5S 3G3, Canada

${ }^{2}$ Yue Kuen Kwok is in the Department of Mathematics, Hong Kong University of Science and Technology, Clear Water Bay, Hong Kong, China

${ }^{3}$ Correspondence author, Department of Mathematics, Hong Kong University of Science and Technology, Hong Kong; fax number: (852)-2358-1643; e-mail: maykwok@ust.hk.
} 
value thus depends on the two stochastic state variables, namely, the interest rate and the path dependent variable of running coupon sum.

In this paper, we construct the pricing model of a typical target redemption note. We observe an analogy of our pricing model with that of an Asian barrier option, whose knock-out barrier is governed by the average asset value (Zvan et al., 1999). When there is only one coupon payment date, we manage to obtain a closed form valuation formula for the note value. When the number of coupon dates is more than one, numerical valuation of the note value is done using numerical partial differential equation methods or Monte Carlo simulation. Under the assumption of one-factor short rate model, we derive the numerical algorithm using the finite volume approach (Zvan et al., 2001). The upstream weighting technique (Vetzal, 1998) is adopted to avoid spurious oscillations in the numerical calculations. We also illustrate how to apply the Monte Carlo simulation method to price the note when the short rate is assumed to follow a two-factor model. Pricing behaviors of the target redemption note under different contract designs and parameter values in the interest rate process are explored. The last section contains a summary and concluding remarks.

\section{FORMULATION OF PRICING MODEL}

The target redemption note is subject to randomness appearing in the cash flow stream due to interest rate fluctuation and potential pre-mature knock-out. The note holder is not certain on the coupon amount received on each coupon date and the termination date at which the par is received. The target redemption note can be considered as a contingent claim with interest rate and path dependent accumulated coupons as the underlying stochastic state variables. We use the short rate $r(t)$ as the interest rate state variable. In this section, we consider the one-factor model of the short rate that follows a stochastic process of the form

$$
d r=\mu(r, t) d t+\bar{\sigma} r^{\beta} d Z,
$$

where $\mu(r, t)$ is the instantaneous drift, $\bar{\sigma} r^{\beta}$ is the instantaneous volatility, and $d Z$ is the differential of a Wiener process. Here, $\bar{\sigma}$ and $\beta$ are constants. When $\mu(r, t)$ is specified to be mean-reverting, then $\beta=0$ and $\beta=1 / 2$ correspond to the well known Vasicek model (1977) and Cox-Ingersoll-Ross (CIR) model (1985), respectively. First, we would like to devise the governing partial differential equation for the value of the target redemption note under the one-factor model. We also derive the closed form price formula of the note when there is only one intermediate coupon date. Pricing of the note under a two-factor short rate model will be considered in a later section.

Let $t_{k}$ denote the $k^{\text {th }}$ coupon date, $k=1,2, \cdots, K$, where $t_{K}=T$ is the note's maturity date. Let $\widehat{N}$ denote the notional value of the note and $\widehat{\tau}$ denote the constant time period between consecutive coupon dates. In the contractual design of the commonly traded target redemption notes, conditional on non-termination of the note at $t_{k}$, the inverse floater formula for the coupon amount received at $t_{k}$ takes the form: $c\left(r, t_{k}\right)=\widehat{N} \widehat{\tau} \max \left(f-m L\left(r, t_{k} ; \widehat{\tau}\right), 0\right)$. Here, $f$ is a constant cap value, $m$ is a constant positive multiplier, $L\left(r, t_{k} ; \widehat{\tau}\right)$ is the $\widehat{\tau}$-period interest rate index (say, LIBOR) at time $t_{k}$. We use $A(t)$ to denote the running sum of coupons received by the holder up to time $t$. Provided that the note survives up to the coupon date $t_{j}$, we have

$$
\begin{array}{ll}
A(t)=\widehat{N} \widehat{\tau} \sum_{k=1}^{j} \max \left(f-m L\left(r, t_{k} ; \widehat{\tau}\right), 0\right) & \text { for } t_{j}^{+}<t<t_{j+1}^{-}, j=1,2, \cdots, K-1, \\
A(t)=0 & \text { for } t<t_{1} .
\end{array}
$$


Here, $t_{j}^{+}$and $t_{j+1}^{-}$denote the time immediately after $t_{j}$ and immediately before $t_{j+1}$, respectively. Let $c_{c a p}$ denote the target cap of the coupon (as percentage of the notional). The guaranteed total sum of coupons received by the holder is $\widehat{N} c_{c a p}$, so $A(t)$ is bounded from above by $\widehat{N} c_{c a p}$. The note is terminated when the total sum of coupons received reaches $\widehat{N} c_{c a p}$. Suppose the note is terminated at $t_{k}$, then the coupon amount received on the termination date is $\widehat{N} c_{c a p}-A\left(t_{k}^{-}\right)$. Let $c\left(r, t_{k}\right)$ denote the coupon amount received at $t_{k}, k=1,2, \cdots, K$, then

$$
c\left(r, t_{k}\right)=\left\{\begin{array}{ll}
\widehat{N} \widehat{\tau} \max \left(f-m L\left(r, t_{k} ; \widehat{\tau}\right), 0\right) & \text { if the note is not terminated at } t_{k} \\
\widehat{N} c_{c a p}-A\left(t_{k}^{-}\right) & \text {if the note is terminated at } t_{k}
\end{array} .\right.
$$

Let $P_{t}^{T}$ denote the value of the unit-par discount bond at time $t$ with maturity date $T$. For the $\widehat{\tau}$-period LIBOR $L(r, t ; \widehat{\tau})$, its value is related to the value of the discount bond value with maturity date $t+\widehat{\tau}$ by

$$
L(r, t ; \widehat{\tau})=\frac{1}{\widehat{\tau}}\left(\frac{1}{P_{t}^{t+\widehat{\tau}}}-1\right) .
$$

For the one-factor Vasicek or CIR model, the analytic solution of $P_{t}^{T}$ can be expressed as

$$
P_{t}^{T}=\exp \left(-B_{1}(T-t) r(t)-B_{0}(t, T-t)\right),
$$

where the solution to $B_{1}(T-t)$ and $B_{0}(t, T-t)$ can be readily obtained (Vasicek, 1977; Cox et al., 1985). In our subsequent exposition, for simplicity sake, we take the notional value $\widehat{N}$ to be one.

\section{Partial differential equation formulation}

Let $V(r, t)$ denote the value of the target redemption note, with dependence on short rate $r$ and calendar time $t$. Since the running sum of coupons $A$ changes only on the coupon dates, so there is no dependence on $A$ in the governing differential equation. Apart from the coupon dates, the note value $V$ is obtained by solving the following partial differential equation.

$$
\frac{\partial V}{\partial t}+\frac{\bar{\sigma}^{2}}{2} r^{2 \beta} \frac{\partial^{2} V}{\partial r^{2}}+\widehat{\mu}(r, t) \frac{\partial V}{\partial r}-r V=0, \quad t_{j}^{+}<t<t_{j+1}^{-},
$$

where

$$
\widehat{\mu}(r, t)=\mu(r, t)+\bar{\sigma} r^{\beta} \lambda(r, t) .
$$

Here, $\lambda(r, t)$ denotes the market price of interest rate risk. On the coupon date $t_{k}, A$ changes according to the updating rule

$$
A\left(t_{k}^{+}\right)=A\left(t_{k}^{-}\right)+c\left(r, t_{k}\right),
$$

where $A\left(t_{k}^{+}\right)$and $A\left(t_{k}^{-}\right)$denote the value of $A$ immediately after and before the coupon date $t_{k}$, respectively. When $A\left(t_{k}^{+}\right)=c_{c a p}$, the note is terminated at $t_{k}^{+}$. The final payout is the sum of the par and the remaining coupon of amount $c_{c a p}-A\left(t_{k}^{-}\right)$. Assuming no premature knock-out at $t_{k}^{+}$, the note value then changes according to the no-arbitrage jump condition

$$
V\left(r, t_{k}^{+}\right)=V\left(r, t_{k}^{-}\right)-c\left(r, t_{k}\right) \quad \text { provided that } A\left(t_{k}^{+}\right)<c_{c a p} .
$$

The note value is evaluated by solving a coupled set of partial differential equations indexed by different values of the path dependent variable $A$ and applying the jump conditions as specified by Eq. (8) on those coupon dates. 
The terminal payoff depends on $A\left(T^{-}\right)$, since the remaining portion of the total guaranteed coupon amount is paid at maturity if no prior knock out occurs. Hence, at time right before expiry, the note value is given by

$$
V\left(r, T^{-}\right)=\left(1+c_{c a p}\right)-A\left(T^{-}\right), \quad \text { if } \quad A\left(T^{-}\right)<c_{c a p} .
$$

In a similar manner, knock-out occurs on a coupon payment date $t_{j}$ when $A\left(t_{j}^{+}\right)$reaches $c_{c a p}$ so that the note value right before $t_{j}$ is given by

$$
V\left(r, t_{j}^{-}\right)=\left(1+c_{c a p}\right)-A\left(t_{j}^{-}\right), \quad j=1,2, \cdots, K-1 .
$$

Here, the knock-out barrier in the pricing model of the target redemption note is associated with the path dependent state variable $A$. As $r \rightarrow \infty, V(r, t) \rightarrow 0$, like those of usual bond pricing models. The boundary condition at the other extreme of the short rate can be quite tricky. For the Vasieck model, $r$ is allowed to assume value from $-\infty$ to $\infty$. However, we are mostly interested in the domain where $r>0$. We are not supposed to impose any boundary condition at $r=0$. Rather, the differential equation for the note value remains valid at $r=0$. Ironically, the note value tends to infinity as $r \rightarrow-\infty$. As for theoretical interest, we postulate that under the Vasicek model

$$
V(r, t) \sim\left(1+c_{\text {cap }}\right) P_{t}^{t_{1}} \quad \text { as } \quad r \rightarrow-\infty, \quad t<t_{1} .
$$

This is because the target redemption note will be terminated on the first coupon date $t_{1}$, thus it behaves like a discount bond with par $1+c_{c a p}$ and maturity date $t_{1}$. For the CIR model, a boundary condition at $r=0$ may be required for certain parameter values. For the detailed discussion on the prescription of the boundary condition at $r=0^{+}$for the CIR model, see the paper by d'Halluin et al. (2001).

\section{Analytic representation}

The value of the target redemption note is given by the sum of present values of all future cash flows. Let $A_{k}, k=1,2, \cdots, K$, denote the set of random variables defined by

$$
A_{k}=\widehat{\tau} \sum_{j=1}^{k} \max (f-m L(r, t ; \widehat{\tau}), 0),
$$

with $A_{0}=0$. By the usual risk neutral valuation approach, we deduce that

$$
\begin{aligned}
V(r, t)= & \sum_{k=1}^{K-1} E_{Q}\left[e ^ { - \int _ { t } ^ { t _ { k } } r ( u ) d u } \left\{\left[\left(1+c_{c a p}\right)-A_{k-1}\right] \mathbf{1}_{\left\{A_{k-1}<c_{c a p} \leq A_{k}\right\}}\right.\right. \\
& \left.\left.+\widehat{\tau} \max (f-m L(r, t ; \widehat{\tau}), 0) \mathbf{1}_{\left\{A_{k}<c_{c a p}\right\}}\right\}\right] \\
& +E_{Q}\left[e^{-\int_{t}^{T} r(u) d u}\left[\left(1+c_{c a p}\right)-A_{K-1}\right] \mathbf{1}_{\left\{A_{K-1}<c_{c a p}\right\}}\right],
\end{aligned}
$$

where $t<t_{1}$ and $E_{Q}$ denotes the expectation under the risk neutral measure $Q$ conditional on $r(t)=r$ and $A(t)=A$. Referring to Eq. (13), the first term represents the scenario in which the target cap has been reached exactly at $t_{k}$ so that the remaining portion of the guaranteed coupon amount $c_{c a p}-A_{k-1}$ and par are paid. The second term shows that coupon of $\widehat{\tau} \max (f-m L(r, t ; \widehat{\tau}), 0)$ is paid when the target cap has not been reached at $t_{k}$. At maturity $T$, the sum of par and remaining coupon amount $\left(1+c_{c a p}\right)-A_{K-1}$ is paid only if knock-out has not occurred, that is, $A_{K-1}<c_{c a p}$. 
When there is only one intermediate coupon date, that is, $K=2$, it is possible to derive the closed form expression for the note value $V$. In this case, Eq. (13) reduces to the following form

$$
\begin{aligned}
V= & E_{Q}\left[e^{-\int_{t}^{t_{1}} r(u) d u}\left[\mathbf{1}_{\left\{A_{1} \geq c_{c a p}\right\}}+\min \left(c_{c a p}, A_{1}\right)\right]\right. \\
& \left.+e^{-\int_{t}^{T} r(u) d u}\left[\mathbf{1}_{\left\{A_{1}<c_{c a p}\right\}}+\max \left(c_{c a p}-A_{1}, 0\right)\right]\right], \quad t<t_{1} .
\end{aligned}
$$

Let $Q_{t_{1}}$ and $Q_{T}$ denote the forward measure with respect to $t_{1}$ and $T$, respectively. The above expression can be simplified as follows

$$
\begin{aligned}
V= & \left(1+c_{c a p}\right) P_{t}^{t_{1}} Q_{t_{1}}\left[A_{1} \geq c_{c a p}\right]+P_{t}^{t_{1}} E_{Q_{t_{1}}}\left[A_{1} \mathbf{1}_{\left\{A_{1}<c_{c a p}\right\}}\right] \\
& +P_{t}^{T} Q_{T}\left[A_{1}<c_{c a p}\right]+P_{t}^{T} E_{Q_{T}}\left[\left(c_{c a p}-A_{1}\right) \mathbf{1}_{\left\{A_{1}<c_{c a p}\right\}}\right], \quad t<t_{1} .
\end{aligned}
$$

Suppose we take $r(t)$ to follow the one-factor Gaussian term structure model, by performing the expectation calculations, we obtain

$$
\begin{aligned}
V=P_{t}^{t_{1}} & \left\{\left(1+c_{c a p}-f \widehat{\tau}-m\right) N\left(\frac{K_{1}-\bar{X}\left(t_{1}\right)}{s}\right)+(f \widehat{\tau}+m) N\left(\frac{K_{2}-\bar{X}\left(t_{1}\right)}{s}\right)\right. \\
& \left.-m e^{\bar{X}\left(t_{1}\right)+s^{2} / 2}\left[N\left(\frac{K_{2}-\bar{X}\left(t_{1}\right)-s^{2}}{s}\right)-N\left(\frac{K_{1}-\bar{X}\left(t_{1}\right)-s^{2}}{s}\right)\right]\right\} \\
+ & P_{t}^{T}\left\{\left(1+c_{c a p}-f \widehat{\tau}-m\right) N\left(\frac{\bar{X}(T)-K_{1}}{s}\right)+(f \widehat{\tau}+m) N\left(\frac{\bar{X}(T)-K_{2}}{s}\right)\right. \\
& \left.-m e^{\bar{X}(T)+s^{2} / 2}\left[N\left(\frac{s^{2}+\bar{X}(T)-K_{1}}{s}\right)-N\left(\frac{s^{2}+\bar{X}(T)-K_{2}}{s}\right)\right]\right\},
\end{aligned}
$$

where $N(\cdot)$ is the cumulative normal distribution function, and

$$
\begin{aligned}
& s^{2}=B_{1}(\widehat{\tau}) \operatorname{var}\left(r\left(t_{1}\right)\right) \\
& K_{1}=\ln \left(\max \left(1+\frac{f \widehat{\tau}-c_{c a p}}{m}, 0\right)\right) \quad \text { and } \quad K_{2}=\ln \left(1+\frac{f \widehat{\tau}}{m}\right) .
\end{aligned}
$$

The derivation of the closed form valuation formula $(16 \mathrm{a}, \mathrm{b})$ is relegated to the Appendix.

\section{FINITE VOLUME SCHEMES UNDER ONE-FACTOR SHORT RATE MODEL}

In this section, we illustrate how to derive the numerical scheme using the finite volume approach for pricing the target redemption note under the one-factor Vasicek or CIR model. The major drawback of a one-factor interest rate model is that this would imply perfect correlation of all LIBOR rates on all coupon dates. When the number of risk factors in the interest rate dynamics is more than one, the Monte Carlo simulation approach exhibits better computational efficiency over the finite volume scheme. The discussion of Monte Carlo simulation calculations under the two-factor short rate model is relegated to the next section.

For the target redemption note, the coupon payments and possible knock-out occur at discrete time instants. We follow the finite volume approach developed by Zvan et al. (2001) for pricing the class of 
discretely monitored path dependent options. The pricing algorithm can be considered as a collection of sub-problems of bond valuation, where each sub-problem is associated with a given value of the path dependent state variable. Here, the path dependent variable is the running sum of coupon payments received. Communication between these independent sub-problems occurs only at the coupon payment dates.

Our proposed finite volume scheme consists of the following three major components:

1. Use of the finite volume approach (Zvan et al., 2001) to derive the discretized equation. Here, the discretized equation at a grid point is obtained by numerical approximation of the integral form of the governing equation over a finite volume domain.

2. Adoption of the upstream weighting technique so that numerical solutions are free of oscillations.

3. Design of the linear interpolation procedure for handling the jump conditions at coupon dates.

Suppose the one-factor CIR interest rate model is used, that is, the risk neutral drift $\widehat{\mu}(r, t)=\alpha-\gamma r+$ $\bar{\sigma} r^{\beta} \lambda(r, t)$ and $\beta=1 / 2$. Here, we use time to expiry $\tau=T-t$ as the temporal variable so that the note value function is written as $V(r, \tau ; A)$. For $T-t_{k}<\tau<T-t_{k-1}, k=1,2, \cdots, K$, we have

$$
\frac{\partial V}{\partial \tau}=\frac{\bar{\sigma}^{2} r}{2} \frac{\partial^{2} V}{\partial r^{2}}+\widehat{\mu}(r, t) \frac{\partial V}{\partial r}-r V, \quad r>0 \text { and } 0 \leq A<c_{c a p},
$$

with auxiliary conditions:

$$
V\left(r, 0^{+} ; A\right)=\left(1+c_{c a p}\right)-A
$$

and at $A=c_{c a p}$

$$
V(r, \tau)=1 .
$$

For a given value of $A$, each one-dimensional sub-problem within the time period between two successive coupon payment dates can be solved independently. We follow the finite volume approach developed by Zvan et al. (2001) for the construction of the numerical scheme. The computational domain is characterized by the grid point $\left(\tau_{n}, A_{i}, r_{j}\right)$, for $n=0,1, \cdots, n_{\max }, i=0,1, \cdots, i_{\max }, j=0,1, \cdots, j_{\max }$. For notational convenience, we set $\tau_{0}=0, r_{0}=0, A_{0}=0$ and $A_{i_{\max }}=c_{c a p}$. We adopt non-uniform grid sizes so that finer grids are used near the maturity time $\tau=0$ and near the current values and boundary values of $A$ and $r$. Let $V_{i, j}^{n}$ denote the numerical solution to the note value at the grid point $\left(\tau_{n}, A_{i}, r_{j}\right)$. Note that for each fixed $i$, the initial condition is posed as $V_{i, j}^{0}=\left(1+c_{c a p}\right)-A_{i}$, for all $j$.

Consider the control volume over the interval $\left[r_{j-1 / 2}, r_{j+1 / 2}\right]$, where $r_{j-1 / 2}$ is the mid-point between $r_{j-1}$ and $r_{j}$, and similar notation for $r_{j+\frac{1}{2}}$. Integrating Eq. (17) over the finite volume (corresponding to the interval $\left.\left[r_{j-1 / 2}, r_{j+1 / 2}\right]\right)$ and approximating the corresponding integrals, we obtain the following implicit scheme:

$$
\begin{aligned}
\frac{V_{i, j}^{n+1}-V_{i, j}^{n}}{\Delta \tau_{n}}= & \frac{\bar{\sigma}^{2} r_{j}}{2 \ell_{j}}\left(\frac{V_{i, j+1}^{n+1}-V_{i, j}^{n+1}}{\Delta r_{j}}-\frac{V_{i, j}^{n+1}-V_{i, j-1}^{n+1}}{\Delta r_{j-1}}\right) \\
& +\frac{\widehat{\mu}\left(r_{j}\right)}{\ell_{j}}\left(V_{i, j+1 / 2}^{n+1}-V_{i, j-1 / 2}^{n+1}\right)-r_{j} V_{i, j}^{n+1}
\end{aligned}
$$

where $\ell_{j}=r_{j+1 / 2}-r_{j-1 / 2}$ denotes the length of the control volume and $\Delta r_{j}=r_{j+1}-r_{j}$ is the step width. The values of $V_{i, j+1 / 2}^{n+1}$ and $V_{i, j-1 / 2}^{n+1}$ in the drift term in Eq. (19) are computed according to the upstream weighting scheme presented below [see Eq. (20) below]. 
When the first-order hyperbolic convective term is large compared to the parabolic diffusion term, the equation is said to be convection dominated. Under dominated convection condition, the numerical solution may suffer from spurious oscillations. In this case, it becomes highly inaccurate to compute the comparative statics such as delta and gamma. To avoid spurious oscillations in the numerical calculations, the upstream weighting scheme is used to compute the value of $V_{i, j+1 / 2}^{n+1}$, which is defined by

$$
V_{i, j+1 / 2}^{n+1}=\left\{\begin{array}{ll}
V_{i, j}^{n+1} & \text { if } \widehat{\mu}\left(r_{j}\right)<0 \\
V_{i, j+1}^{n+1} & \text { if } \widehat{\mu}\left(r_{j}\right) \geq 0
\end{array} .\right.
$$

The initial and boundary conditions are given by

$$
\begin{array}{ll}
V_{i, j}^{0}=1+c_{\text {cap }}-A_{i} & \text { for all } i \text { and } j \\
V_{i_{\text {max }}, j}^{n}=1 & \text { for all } n \text { and } j \\
V_{i, j_{\text {max }}}^{n}=0 & \text { for } n \neq 0, i \neq i_{\text {max }} .
\end{array}
$$

More generally, one may assign a temporal weighting factor $\theta, 0 \leq \theta \leq 1$, to the spatial discretization terms evaluated at the new and old time levels. This leads to the following weighted finite volume scheme:

$$
\begin{aligned}
\frac{V_{i, j}^{n+1}-V_{i, j}^{n}}{\Delta \tau_{n}}= & \theta\left[\frac{\bar{\sigma}^{2} r_{j}}{2 \ell_{j}}\left(\frac{V_{i, j+1}^{n+1}-V_{i, j}^{n+1}}{\Delta r_{j}}-\frac{V_{i, j}^{n+1}-V_{i, j-1}^{n+1}}{\Delta r_{j-1}}\right)\right. \\
& \left.+\frac{\widehat{\mu}\left(r_{j}\right)}{\ell_{j}}\left(V_{i, j+1 / 2}^{n+1}-V_{i, j-1 / 2}^{n+1}\right)-r_{j} V_{i, j}^{n+1}\right] \\
& +(1-\theta)\left[\frac{\bar{\sigma}^{2} r_{j}}{2 \ell_{j}}\left(\frac{V_{i, j+1}^{n}-V_{i, j}^{n}}{\Delta r_{j}}-\frac{V_{i, j}^{n}-V_{i, j-1}^{n}}{\Delta r_{j-1}}\right)\right. \\
& \left.+\frac{\widehat{\mu}\left(r_{j}\right)}{\ell_{j}}\left(V_{i, j+1 / 2}^{n}-V_{i, j-1 / 2}^{n}\right)-r_{j} V_{i, j}^{n}\right] .
\end{aligned}
$$

By setting $\theta=0,1 / 2$ and 1 , we obtain the fully explicit scheme, Crank-Nicolson scheme and fully implicit scheme, respectively.

Two desirable attributes of a numerical scheme are stability and absence of spurious oscillations. With regard to stability, both the Crank-Nicolson and fully implicit schemes are unconditionally stable. The fully explicit scheme is stable only if the time step is sufficiently small relative to the spatial grid size. The advantage of the Crank-Nicolson scheme is that it is second order accurate in time while both implicit and explicit schemes exhibit only linear rate of convergence. With regard to oscillations, fully implicit scheme is not susceptible to spurious oscillations while the Crank-Nicolson and explicit schemes require certain time step constraint in order to avoid numerical oscillations.

\section{Jump conditions on coupon dates}

A jump condition is applied on each coupon date $t_{k}$. In terms of time to expiry, suppose the time to expiry corresponding to the coupon date $t_{k}$ falls between the two time levels $\tau_{n}$ and $\tau_{n+1}$, where $\tau_{n} \leq T-t_{k}<\tau_{n+1}$, the finite volume scheme is modified as shown below

$$
V_{i, j}^{n+1}=\widehat{V}_{i, j}^{n}+\min \left\{c_{c a p}-A_{i}, \widehat{\tau} \max \left(f-m L\left(r_{j}, t_{k} ; \widehat{\tau}\right), 0\right)\right\}
$$


Here, $\widehat{V}_{i, j}^{n}$ is the approximate value of $V\left(r_{j}, \tau_{n} ; A^{\prime}\right)$ computed by using the following linear interpolation procedure (Forsyth et al., 2002):

$$
\widehat{V}_{i, j}^{n}=\frac{A^{\prime}-A_{i^{\prime}-1}}{A_{i^{\prime}}-A_{i^{\prime}-1}} V_{i^{\prime}, j}^{n}+\frac{A_{i^{\prime}}-A^{\prime}}{A_{i^{\prime}}-A_{i^{\prime}-1}} V_{i^{\prime}-1, j}^{n},
$$

where

$$
A^{\prime}=A_{i}+\min \left\{c_{c a p}-A_{i}, \widehat{\tau} \max \left(f-m L\left(r_{j}, t_{k} ; \widehat{\tau}\right), 0\right)\right\} .
$$

Normally, $A^{\prime}$ does not fall onto one of the computational grid points and so linear interpolation is performed to estimate $V\left(r_{j}, \tau_{n} ; A^{\prime}\right)$ using $V_{i^{\prime}, j}^{n}$ and $V_{i^{\prime}-1, j}^{n}$, where $A^{\prime}$ lies between $A_{i^{\prime}-1}$ and $A_{i^{\prime}}$. The second term in $A^{\prime}$ is the actual coupon payment at time $t=t_{k}$. Note that $A^{\prime}$ is capped by $c_{c a p}$ since the note holder receives at most the accumulated coupon $c_{c a p}$. In this way, $A^{\prime}$ always falls between 0 and $c_{\text {cap }}$. A schematic representation of the linear interpolation procedure is illustrated in Figure 1.

\section{MONTE CARLO SIMULATION UNDER TWO-FACTOR SHORT RATE MODEL}

The value of a target redemption note $V$ is given by the expectation under the risk neutral measure $Q$ of the future cash flows as defined in Eq. (14). Following the Monte Carlo simulation approach, $V$ can be approximated by taking the average of the simulated values of $Y$, where $Y$ is the sum of discounted cash flows received by the note holder. Note that $Y$ involves LIBOR $L\left(r, t_{k} ; \widehat{\tau}\right)$, cumulative coupon $A_{k}$ and the date of redemption of the par. Given the realization of the short rate process $r$, one can obtain $Y$ through the evaluation of $L\left(r, t_{k} ; \widehat{\tau}\right), A_{k}$ and $t^{*}$, where $t^{*}$ is the date of redemption. Suppose a two-factor interest rate model is used, the dynamics of a random vector $\boldsymbol{x}=\left(x_{1} x_{2}\right)^{T}$ is assumed to be governed by the process:

$$
d \boldsymbol{x}=\boldsymbol{\mu}(\boldsymbol{x}, t) d t+\sigma(\boldsymbol{x}, t) d \boldsymbol{Z},
$$

where $\boldsymbol{Z}=\left(Z_{1} Z_{2}\right)^{T}$ and $Z_{1}$ and $Z_{2}$ are two independent Wiener processes under $Q$. In our calculations, we use the two-factor Gaussian model where

$$
\boldsymbol{\mu}(\boldsymbol{x}, t)=\left(\begin{array}{l}
-\kappa_{1} \boldsymbol{x}_{1} \\
-\kappa_{2} \boldsymbol{x}_{2}
\end{array}\right), \quad \sigma(\boldsymbol{x}, t)=\left(\begin{array}{cc}
\sigma_{1} & 0 \\
\sigma_{2} \rho & \sigma_{2} \sqrt{1-\rho^{2}}
\end{array}\right),
$$

the short rate is given by

$$
r(t)=x_{1}(t)+x_{2}(t)+b(t) .
$$

Here, $\kappa_{i}$ and $\sigma_{i}$ are the reversion rate and the volatility parameter of the process $x_{i}, i=1,2$, and $\rho$ is the correlation coefficient between the risk factors $x_{1}$ and $x_{2}$. Also, $b(t)$ is a deterministic time dependent function which fits the current term structure. Based on the dynamic of $\boldsymbol{x}$, the computational steps of simulating $Y$ using Monte Carlo simulation are summarized as follows:

1. Initially, set $k=1, A=0, Y=0$ and $x=x_{0}$. Here, $x_{0}$ depends on the current interest rate and the choice of the short-rate model.

2. Simulate the path of $x(t)$ from $t=t_{k-1}$ to $t=t_{k}$. Observe that $r$ is a function of $x$ and $t$.

3. Compute the actual coupon, $c=\min \left\{c_{c a p}-A, \widehat{\tau} \max (f-m L, 0)\right.$, where the LIBOR $L$ is a function of $t_{k}$ and $x\left(t_{k}\right)$. 
4. Update $Y, A$ and $k$ by $Y+c, A+c$ and $k+1$, respectively.

5. If $A=c_{c a p}$ or $k>K$, then update $Y$ by $Y+\left[\left(1+c_{c a p}\right)-A\right]$ and terminate the procedure. Otherwise, go to Step 2.

\section{SAMPLE CALCULATIONS}

We performed sample calculations to illustrate the numerical performance of the proposed finite volume scheme and Monte Carlo simulation for pricing various forms of target redemption notes. We examine the pricing behaviors of target redemption notes subject to varying values of interest rate level, volatility of interest rate, reversion level and reversion speed in the interest rate dynamics. We also consider the probability of premature termination of the note at different coupon dates under varying values of the correlation coefficient between the risk factors. In addition, we investigate how the note value varies with different inverse floater formulas. Throughout this section, except otherwise specified, the terms in the contract of the target redemption note used in our sample calculations are specified as follows:

$\begin{array}{ll}\text { Notional amount } & 100 \\ \text { Target cap rate } & 15 \% \\ \text { First year coupon rate (fixed) } & 9 \% \\ \text { Inverse floater formula } & \max (8.5 \%-2 L, 0), L=3 \text {-month LIBOR } \\ \text { Coupon payment frequency } & \text { quarterly } \\ \text { Maturity date } & 5 \text { years from now }\end{array}$

One-factor model

First, we price the target redemption note under a one-factor short rate model and compare the numerical results obtained from the implicit finite volume scheme and Monte Carlo simulation. The risk neutral dynamics of the short rate under the CIR model is assumed to be

$$
d r=[\gamma(r-\alpha)+\lambda r] d t+\bar{\sigma} r^{1 / 2} d Z,
$$

where the market price of risk is specified as $\frac{\lambda r^{1 / 2}}{\bar{\sigma}}$ so that the adjustment term $\lambda r$ is added to the drift term to give the risk neutral dirft $\gamma(r-\alpha)+\lambda r$ in the CIR model. In our sample calculations, the parameter values in the CIR interest rate model are taken to be: $\alpha=0.02, \gamma=0.5, \lambda=0.01$ and $\bar{\sigma}=0.2$. For the construction of the discretized computational domain, we use 60 grids along the $r$-direction and 15 grids along the $A$-direction, and $r_{\max }$ is taken to be 0.9 .

In Figure 2, we show the comparison of accuracy of numerical valuation of the target redemption note using the finite volume method with varying number of time steps. The convergence trend of the numerical results with increasing number of time steps is observed. Also, the note value $V$ is seen to be a decreasing function of the interest rate $r$, which drops quite significantly with increasing value of $r$. This is due to the nature of the inverse floater formula in calculating the coupon accumulation. A higher interest rate implies that coupons are received at a slower rate and consequently a lower chance of premature knock-out. With delay of termination of the note, the par would be discounted at a higher discount factor so there is a drop in the note value.

In Table 1, we list the numerical values of the target redemption note at different current interest rate levels obtained from the finite volume method and Monte Carlo simulation method. We do observe good 
agreement of the numerical results obtained from both numerical methods, with percentage difference typically less that $0.5 \%$ when the number of time steps is 120 and number of simulation runs is 10,000 . The observed standard errors with 10,000 simulation runs are typically less than $1 \%$.

We would like to examine the sensitivity of the note value $V$ on the interest rate volatility parameter $\bar{\sigma}$ under the one-factor CIR interest rate model. In Figure 3, we plot the note value $V$ against the short rate $r$ with varying values of $\bar{\sigma}$. It is observed that higher interest rate volatility leads to higher note value but the sensitivity of $V$ on $\bar{\sigma}$ is not quite significant. Also, we would like to examine the impact of the reversion level $\alpha$ and reversion speed $\gamma$ on the note value. In Figures 4 and 5, we show the plot of note value $V$ against $\alpha$ and $\gamma$, respectively, with varying values of the short rate $r$. It is seen that $V$ is a decreasing function of $\alpha$ but an increasing function of $\gamma$. Interestingly, the note value exhibits quite high sensitivity on $\alpha$. The decreasing property of the note value with respect to $\alpha$ is attributed to higher future interest rates. This would lead to higher discount factors and smaller chance of pre-mature knock-out. The impact of the parameter $\gamma$ on the note value has somewhat opposite effect as that of $\alpha$ since these two parameters have opposite signs in the drift term of the CIR model, thus the note value $V$ is an increasing function of $\gamma$.

\section{Two-factor model}

Under the two-factor Gaussian short rate model as defined in Eqs. (25-27), we are interested in finding the probability (under the risk neutral measure) of premature redemption of the note on different coupon dates under varying values of the correlation coefficient of risk factors $\rho$. In Table 2 , we tabulate the calculated values of risk neutral probability of pre-mature redemption with $\rho=-0.5,0,0.5$. Other parameter values of the two-factor Gaussian interest rate model used in the Monte Carlo calculations are: $\kappa_{1}=0.7, \sigma_{1}=0.08, \kappa_{2}=0.1$ and $\sigma_{2}=0.05$. The initial yield curve is chosen to be $Y_{0}(T)=$ $0.07-0.04 e^{-0.2 T}$. When $\rho$ becomes more negative, the LIBOR rates on the coupon dates have lower correlation. At the current low interest rate of $1.5 \%$, the probability of early redemption in the $4^{\text {th }}$ year is higher since the chance of remaining at low interest rate level is smaller.

Lastly, we show how the contract specification of the inverse floater formula influences the note value. We consider 10 cases of contract specification of the target redemption note with varying maturities. All these contracts have fixed coupon rate of $9 \%$ in the first year and the target coupon rate is $15 \%$. In Table 3, we list the values of these sample target redemption notes. The note values are computed using the two-factor Gaussian interest rate model with parameter values: $r=1.5 \%, \kappa_{1}=0.7, \sigma_{1}=0.08, \kappa_{2}=$ $0.1, \sigma_{2}=0.05$ and $\rho=0.5$. For the two notes labeled as Sample 4 and Sample 7 , they have almost identical contract specification except with differing maturity dates. We observe that the note with longer time to expiry has a lower value. A major contributing factor may be due to the longer waiting time for receiving the par payment. Sample 5 and Sample 10 are almost identical except that the second year coupon rate is fixed at $3 \%$. A fixed coupon paid with certainty in the second year leads to a higher chance of meeting the target level earlier in subsequent years, so it has a higher value compared to that of Sample 5.

\section{CONCLUSION}

The intrinsic difficulties in pricing the target redemption note arise from the uncertainty in the date of redemption of the note since the note will be terminated on the coupon date when the accumulated coupon received by the holder has reached the pre-set target sum. In this paper, we construct the pricing models of the target redemption note by modeling the interest rate uncertainty using both one-factor and two-factor short rate models. Similar to the Asian barrier option, the knock-out feature of the note 
is determined by a path dependent variable which is defined by the running sum of accumulated coupon received. For the simplified case where there is only one coupon date, we obtain closed form price formula of the note value.

When the interest rate dynamics is governed by a one-factor model, we devise the finite volume scheme for numerical valuation of the pricing model. We employ the upstream weighting technique in order to avoid excessive oscillations in the numerical calculations. Also, the linear interpolation technique is used to deal with the jump condition across a coupon payment date. We use Monte Carlo simulation method for pricing the note when the number of risk factors in the interest rate dynamics is taken to be two. Numerical accuracy of the finite volume and Monte Carlo algorithms for pricing target redemption notes were tested and good agreements of the numerical results are observed.

Our sample calculations reveal that the note value is highly sensitive on the interest rate level but show little dependence on the volatility of interest rate. Based on the one-factor Cox-Ingersoll-Ross mean reversion model, we show that the note value is a decreasing function of the reversion level and an increasing function of the reversion speed. When the interest rate dynamics is governed by the twofactor Gaussian short rate model, we observe that the probability of pre-mature redemption increases with decreasing value of the correlation coefficient among the two risk factors in the two-factor short rate model. Lastly, we also examine how various forms of contract specification, like note's maturity and analytic form of the inverse floater formula influence the probability of pre-mature knock-out, and consequently the note value. When the current interest rate level is low, the note value is not quite sensitive to the contract specification.

\section{References}

[1] Cox, J.C., Ingersoll, J.E. Jr., \& Ross, S.A. (1985). A theory of the term structure of interest rate. Econometrica, 53, 385-407.

[2] D'Halluin, Y., Forsyth, P.A., Vetzal, K.R., \& Labahn, G.. (2001). A numerical PDE approach for pricing callable bonds. Applied Mathematical Finance, 8, 49-77.

[3] Forsyth, P.A., Vetzal, K.R., \& Zvan, R. (2002). Convergence of numerical methods for valuing path dependent options using interpolation. Review of Derivatives Research, 5, 273-314.

[4] Vasicek, O. (1977). An equilibrium characterization of the term structure. Journal of Financial Economics, 5, 177-188.

[5] Vetzal, K.R. (1998). An improved finite difference approach to fitting the initial term structure. Journal of Fixed Income, 7, 62-81.

[6] Zvan, R., Forsyth, P.A., \& Vetzal, K.R. (Fall, 1999). Discrete Asian barrier options. Journal of Computational Finance, 3, 41-68.

[7] Zvan, R., Forsyth, P.A., \& Vetzal, K.R. (2001). A finite volume approach for contingent claims valuation. IMA Journal of Numerical Analysis, 21, 703-731.

\section{APPENDIX - Derivation of price formula $(16 a, b)$}


Suppose the discount bond value $P_{t}^{T}$ assumes the form as given by Eq. (5), we define the random variable

$$
X=B_{0}\left(t_{1}, \widehat{\tau}\right)+B_{1}(\widehat{\tau}) r\left(t_{1}\right)
$$

From Eqs. (4) and (12), we obtain

$$
A_{1}=\max \left(f \widehat{\tau}+m-m e^{X}, 0\right) .
$$

It is easily seen that

$$
\left\{A_{1}<c_{c a p}\right\} \Leftrightarrow\left\{X>\ln \max \left(1+\frac{f \widehat{\tau}-c_{c a p}}{m}, 0\right)\right\} .
$$

We also define the following means and variance

$$
\begin{aligned}
\bar{X}\left(t_{1}\right) & =B_{0}\left(t_{1}, \widehat{\tau}\right)+B_{1}(\widehat{\tau}) E_{Q_{t_{1}}}\left[r\left(t_{1}\right)\right] \\
\bar{X}(T) & =B_{0}\left(t_{1}, \widehat{\tau}\right)+B_{1}(\widehat{\tau}) E_{Q_{T}}\left[r\left(t_{1}\right)\right] \\
s^{2} & =B_{1}(\widehat{\tau}) \operatorname{var}\left(r\left(t_{1}\right)\right) ;
\end{aligned}
$$

and when $r(t)$ is assumed to be Gaussian, we observe that

$$
\begin{aligned}
& X \sim N\left(\bar{X}\left(t_{1}\right), s^{2}\right) \quad \text { under } \quad Q_{t_{1}} \text {, } \\
& X \sim N\left(\bar{X}(T), s^{2}\right) \text { under } Q_{T},
\end{aligned}
$$

where $N\left(\mu, \sigma^{2}\right)$ denote a Gaussian distribution with mean $\mu$ and variance $\sigma^{2}$. It is straightforward to show that

$$
\begin{aligned}
& Q_{t_{1}}\left[A_{1} \geq c_{c a p}\right]=N\left(\frac{K_{1}-\bar{X}\left(t_{1}\right)}{s}\right) \\
& E_{Q_{t_{1}}}\left[A_{1} \mathbf{1}_{\left\{A_{1}<c_{c a p}\right\}}\right]=(f \widehat{\tau}+m)\left[N\left(\frac{K_{2}-\bar{X}\left(t_{1}\right)}{s}\right)-N\left(\frac{K_{1}-\bar{X}\left(t_{1}\right)}{s}\right)\right] \\
& \quad-m e^{\bar{X}\left(t_{1}\right)+s^{2} / 2}\left[N\left(\frac{K_{2}-\bar{X}\left(t_{1}\right)-s^{2}}{s}\right)-N\left(\frac{K_{1}-\bar{X}\left(t_{1}\right)-s^{2}}{s}\right)\right],
\end{aligned}
$$

and similar results for the expectation calculations under $Q_{T}$. 


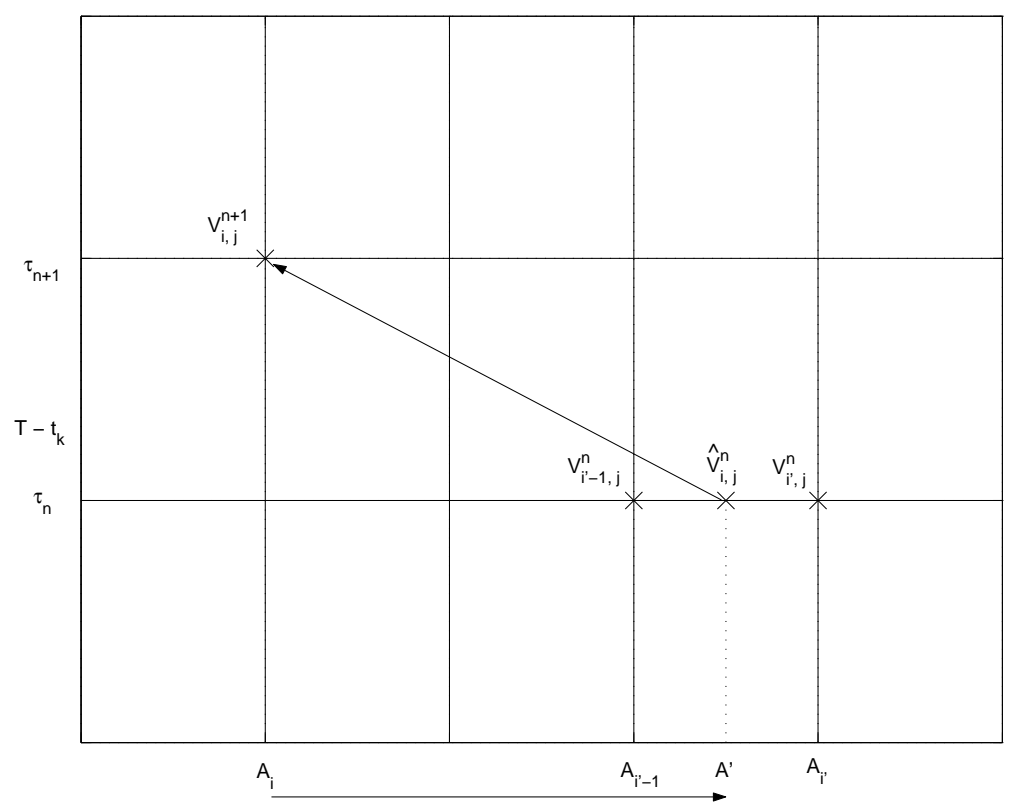

Figure $1 \mathrm{~A}$ schematic representation of the linear interpolation procedure at $\tau_{n}$. Here, $A_{i}$ jumps to $A^{\prime}$ according to Eq. (24b). Normally, $A^{\prime}$ does not fall onto one of the computational grid points so that linear interpolation is performed to estimate $V\left(r_{j}, \tau_{n} ; A^{\prime}\right)$ using $V_{i^{\prime}, j}^{n}$ and $V_{i^{\prime}-1, j}^{n}$ where $A^{\prime}$ lies between $A_{i^{\prime}-1}$ and $A_{i^{\prime}}$ [see Eq. (24a)].

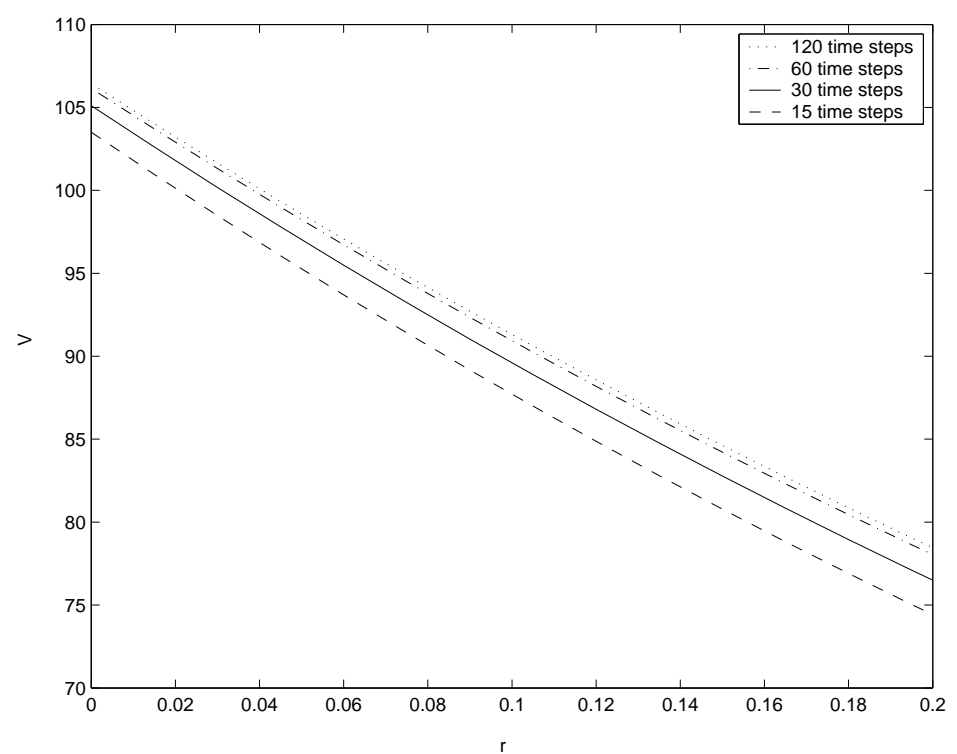

Figure 2 Comparison of accuracy of numerical valuation of the target redemption note using the finite volume scheme with varying number of time steps. 


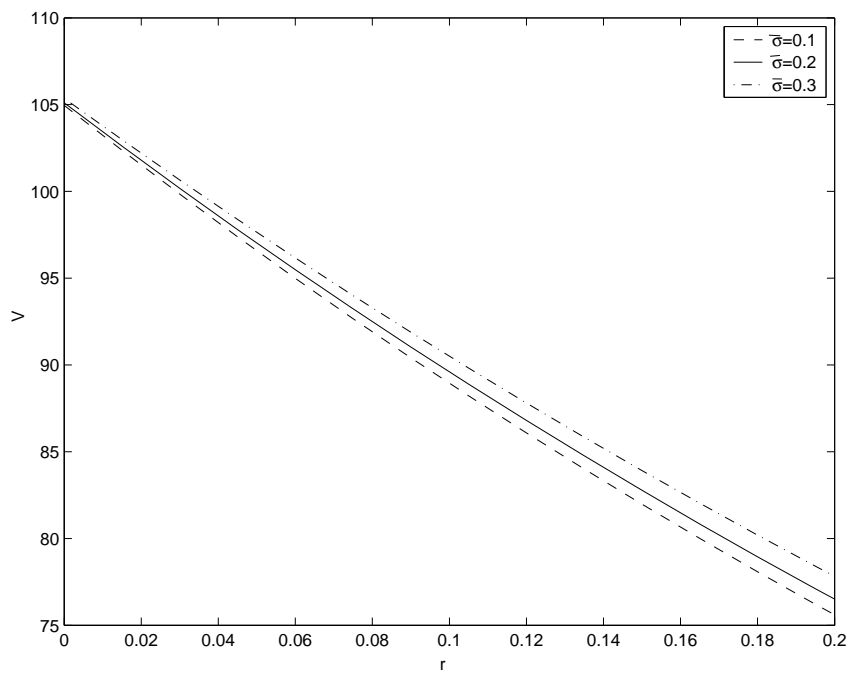

Figure 3 Plot of note value $V$ against short rate $r$ with varying values of volatility parameter $\bar{\sigma}$.

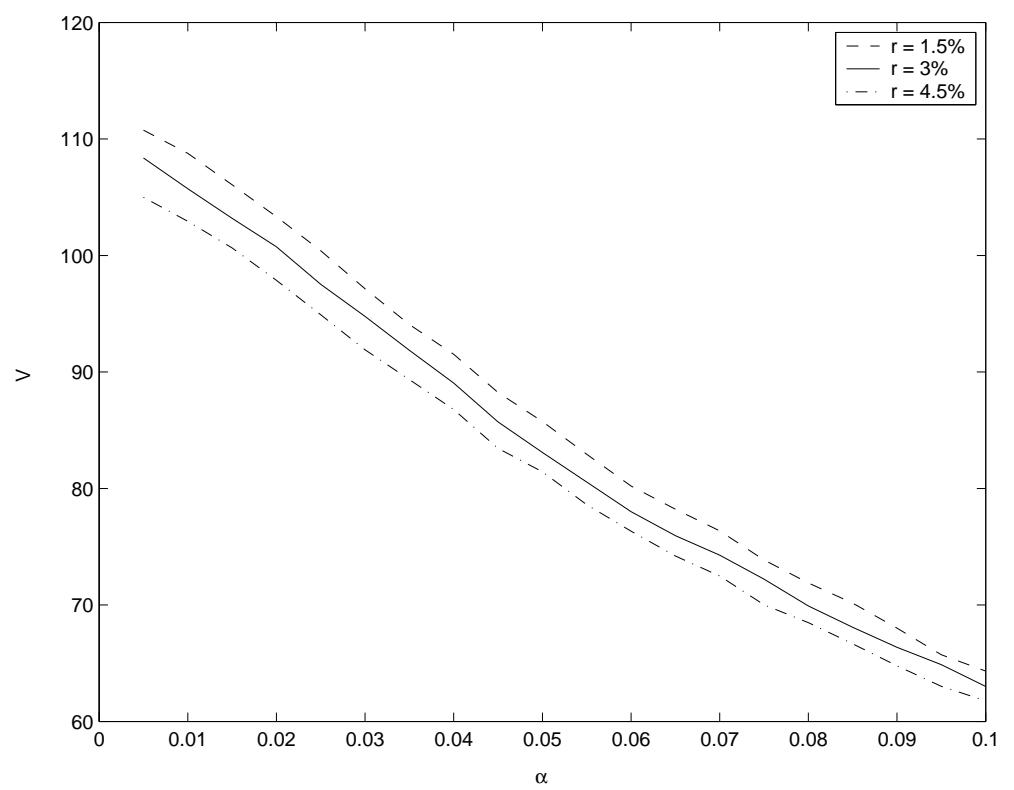

Figure 4 Plot of note value $V$ against reversion level $\alpha$ (as specified in the one-factor CIR model) with varying values of short rate $r$. 


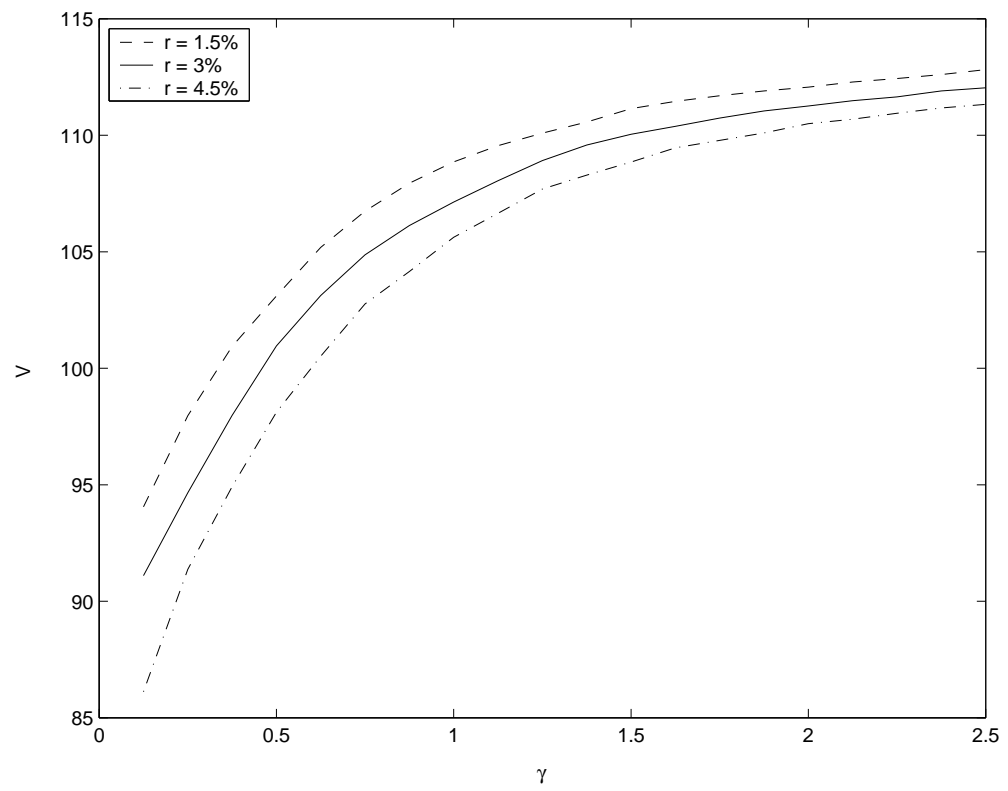

Figure 5 Plot of note value $V$ against reversion speed $\gamma$ (as specified in the one-factor CIR model) with varying values of short rate $r$.

\begin{tabular}{l|l|l|l} 
Current interest rate level & $1.5 \%$ & $3.0 \%$ & $4.5 \%$ \\
\hline Finite volume calculations & & & \\
\hline 15 time steps & 100.96 & 98.47 & 96.06 \\
\hline 30 time steps & 102.61 & 100.18 & 97.80 \\
\hline 60 time steps & 103.71 & 101.32 & 98.99 \\
\hline 120 time steps & 104.02 & 101.65 & 99.33 \\
\hline Monte Carlo simulation & & & \\
\hline 1,000 simulation runs & 103.79 & 100.02 & 97.98 \\
(standard error) & $(3.23)$ & $(3.19)$ & $(3.12)$ \\
\hline 10,000 simulation runs & 103.61 & 100.79 & 98.16 \\
(standard error) & $(1.04)$ & $(1.01)$ & $(0.99)$
\end{tabular}

Table 1 Numerical values of the target redemption note at different current interest rate levels obtained from (i) finite volume calculations using varying number of time steps, (ii) Monte Carlo simulation using varying number of simulation runs. The values inside the brackets are standard errors as observed in the simulation runs. 


\begin{tabular}{c|c|c|c|c|}
$\rho$ & $t_{k}$ & & \\
$1^{\text {st }}$ year & $2^{\text {nd }}$ year & $3^{\text {rd }}$ year & $4^{\text {th }}$ year \\
\hline-0.5 & 0 & 0.0399 & 0.0596 & 0.9005 \\
0 & 0 & 0.0986 & 0.0790 & 0.8224 \\
0.5 & 0 & 0.1413 & 0.0890 & 0.7688
\end{tabular}

Table 2 Probability (under the risk neutral measure) of pre-mature redemption of the note on different coupon dates using varying values of the correlation coefficient $\rho$.

\begin{tabular}{c|l|c} 
Sample & inverse floater formula & note value \\
\hline 1 & 2nd to 4th year : $\max (6 \%-2 L, 0)$ & 104.95 \\
2 & 2nd to 4th year: $\max (8.5 \%-2 L, 0)$ & 105.09 \\
3 & 2nd to 4th year : $\max (10 \%-2 L, 0)$ & 105.47 \\
4 & 2nd to 5th year : $\max (6 \%-2 L, 0)$ & 100.83 \\
5 & 2nd to 5th year : $\max (8.5 \%-2 L, 0)$ & 101.59 \\
6 & 2nd to 5th year : $\max (10 \%-2 L, 0)$ & 101.86 \\
7 & 2nd to 6th year : $\max (6 \%-2 L, 0)$ & 98.01 \\
8 & 2nd to 6th year : $\max (8.5 \%-2 L, 0)$ & 98.75 \\
9 & 2nd to 6th year : $\max (10 \%-2 L, 0)$ & 99.41 \\
10 & 2nd year : fixed at 3\% and & 103.44 \\
& 3rd to 5th year : $\max (8.5 \%-2 L, 0)$ &
\end{tabular}

Table 3 Impact of note maturity and the inverse floater formula in the contract specification on the note value. 